\title{
Relation between tag position and degree of visualized cerebrospinal fluid reflux into the lateral ventricles in time-spatial labeling inversion pulse magnetic resonance imaging at the foramen of Monro
}

\author{
Erik H Middlebrooks ${ }^{1,2^{*}}$, Jeffrey A Bennett ${ }^{1,2}$ and Alissa Old Crow ${ }^{1,2}$
}

\begin{abstract}
Background: Time-spatial labeling inversion pulse (Time-SLIP) magnetic resonance imaging allows non-invasive visualization of cerebrospinal fluid (CSF) movement. Our study evaluated the sensitivity of the Time-SLIP tag placement on the measurement of CSF reflux from the third ventricle into the lateral ventricles via the foramen of Monro.

Findings: Multiple Time-SLIP MRI scans were obtained in three healthy volunteers (23-55 years of age) evaluating the observed CSF pulsation and reflux from the third ventricle into the lateral ventricles while varying the placement of the tag. Linear regression was performed to evaluate the effects of tag position on the amount of visualized reflux and pulsation. Variation in the position of the tag relative to the plane of the free margin of the septum pellucidum produced a significant inverse variation in the observed reflux into the lateral ventricles $\left(R^{2}=0.74\right)$. The further the distance of the top (superior edge) of the tag from the plane of the free margin of the septum pellucidum, the less reflux into the lateral ventricles was observed $(P=0.006)$.
\end{abstract}

Conclusions: The amount of observed CSF reflux into the lateral ventricles in Time-SLIP MR imaging is dependent on the positioning of the CSF tag with decreasing amount of visualized reflux the further caudal the CSF tag is relative to the free margin of the septum pellucidum.

Keywords: Magnetic resonance imaging, Cerebrospinal fluid, Physiology, Spin labels

\section{Findings}

Background

Cerebrospinal fluid (CSF) plays a pivotal role in normal physiology of the human central nervous system (CNS). CSF is thought to perform many functions such as supplying mechanical support for the brain, contributing to maintenance of intracranial pressure, stability against traumatic forces, and in clearance of metabolic by-products $[1,2]$. With an understanding of the normal

\footnotetext{
*Correspondence: ehmiddlebrooks@gmail.com

'Department of Radiology, College of Medicine, University of Florida,

1600 SW Archer Rd., PO Box 100374, Gainesville, FL 32610, USA

Full list of author information is available at the end of the article
}

physiologic role of CSF, it is apparent that abnormalities in CSF function may serve as a basis for underlying diseases of the brain. Previous research has demonstrated impaired CSF flow in many diseases including, but not limited to, normal pressure hydrocephalus (NPH) [3], Alzheimer's disease [4], Chiari malformation [5], and multiple sclerosis [6].

MR sequences such as phase-contrast imaging (PCMRI) have had a substantial impact on our ability to monitor changes in CSF flow [7, 8]. Yamada et al. [8] recently repurposed an arterial spin labeling variant known as time-spatial labeling inversion pulse (TimeSLIP) to noninvasively visualize CSF flow without relying on velocity-dependent phase changes as in PC-MRI. 
Time-SLIP utilizes a nonselective inversion recovery pulse to invert all longitudinal magnetization in the fieldof-view followed by a second spatially-selective inversion pulse (tag pulse). This spatially-selective pulse results in equilibrium magnetization of only the fluid and tissue located within the user-selected tag region. Immediately following the selective tag pulse, the magnetization outside of the tag will begin to relax back towards equilibrium. As the background magnetization approaches the null point, images can be acquired using a single-shot fast spin-echo sequence. Magnetization that originated within the user-selected tag will appear bright whereas magnetization outside of the tag will appear dark. CSF that has traveled outside of the tag during the recovery time will be visualized easily against the dark signal of tissue that did not originate within the tag. Repeated images are then obtained at different recovery times, triggered by cardiac gating with a photoplethysmogram, allowing visualization of CSF displacement [9-11].

The purpose of our study is to evaluate the effects of tag placement on the measurement of CSF reflux from the third ventricle into the lateral ventricles via the foramen of Monro. This normal physiologic phenomenon has been reported to be abnormal in various clinical conditions such as normal pressure hydrocephalus and noncommunicating hydrocephalus $[9,10]$. The results of the study would ideally determine if the tag placement adversely affects reflux measurements and if an optimal position for tag placement exists. This may avert erroneous interpretation of CSF reflux changes due to incorrect tag placement.

\section{Methods}

\section{Subjects}

Our HIPAA-compliant study met criteria for exemption from full review of the University Institutional Review Board. Informed consent was waived for retrospective review of pre-existing data. Three healthy volunteers ranging from 23 to 55 years of age were imaged.

\section{Image acquisition}

Imaging was performed on a Vantage Titan 3T MRI (Toshiba, Tokyo, Japan) with a 32-channel head coil. The Time-SLIP sequence was obtained in an oblique coronal plane oriented parallel to the long-axis of the foramen of Monro (See Additional file 1: Figure S1 MR images showing the selection of imaging plane). The oblique coronal plane was determined by initially obtaining a 3D sagittal localizer to identify the foramen of Monro. The localizer was a 3D fast advanced spin echo (FASE) with a TR of $6,000 \mathrm{~ms}$ and TE of $153 \mathrm{~ms}$. The oblique coronal plane was then selected as the plane through the foramen of Monro which is parallel to the long axis of the foramen.

\section{Sequence parameters}

The Time-SLIP sequence utilized a TR of approximately $13,000 \mathrm{~ms}$ ( $15 \mathrm{R}-\mathrm{R}$ intervals to allow for maximum CSF recovery) and a TE of $80 \mathrm{~ms}$. The matrix size was $224 \times 224$ with a field of view of $23 \times 23 \mathrm{~cm}$ and slice thickness of 5-mm. Electrocardiographic time delay from an $\mathrm{R}$ wave to the initiation of the nonselective inversion recovery pulse was $0 \mathrm{~ms}$. The subsequent 2-D single-shot FSE images were obtained within one acquisition with variable TIs increasing in increments of $200 \mathrm{~ms}$ for a total of 15 times starting at 2,000 ms. The acquisition time for each individual run averaged about 3.5 min depending on pulse rate.

\section{Measurements}

Multiple scans were obtained in each subject with the tag varying in position. The top (superior) of the tag was placed in locations ranging from $8.5 \mathrm{~mm}$ below the free margin of the septum pellucidum (FMSP) to $13 \mathrm{~mm}$ above the FMSP. Images were reviewed at a dedicated radiology imaging workstation. The distance from the top (superior aspect) of the tag and the plane of the FMSP was determined. The plane of the FMSP was defined as a perpendicular line tangential to the inferior-most aspect of the septum pellucidum (see Additional file 2: Figure S2. MR images showing the plane of FMSP and normal reflux). For each acquisition, two additional measurements were made. The first measurement was the greatest cephalad height that the CSF pulsated above the plane of the tagged fluid across all time points. The second measurement was the amount of CSF reflux into the lateral ventricles determined by the height of CSF reflux above the plane of the FMSP (see Additional file 3: Figure S3. Measurement of CSF pulsation.).

\section{Statistical analysis}

Statistical analysis was performed with Prism 6.0 (GraphPad Software, Inc. San Diego, CA, USA). Data was analyzed with a linear regression model to determine correlations between measurements of CSF displacement relative to the placement of the CSF tag. Only those measurements with the tag placed below the plane of the FMSP were used in regression analysis of the reflux height above the plane of the FMSP.

\section{Results}

Variation in the position of the tag relative to the plane of the FMSP produced a significant variation in the measured reflux into the lateral ventricles (Figure 1a). The amount of reflux above the plane of the FMSP was inversely correlated with distance of the tag placement from the plane $\left(y=0.69 x+7.3 ; R^{2}=0.74\right)$. That is, the further the distance of the top (superior edge) of the tag from the plane of the FMSP, the less visualized reflux into the lateral ventricles $(P=0.006)$. Analysis of each individual subject also 

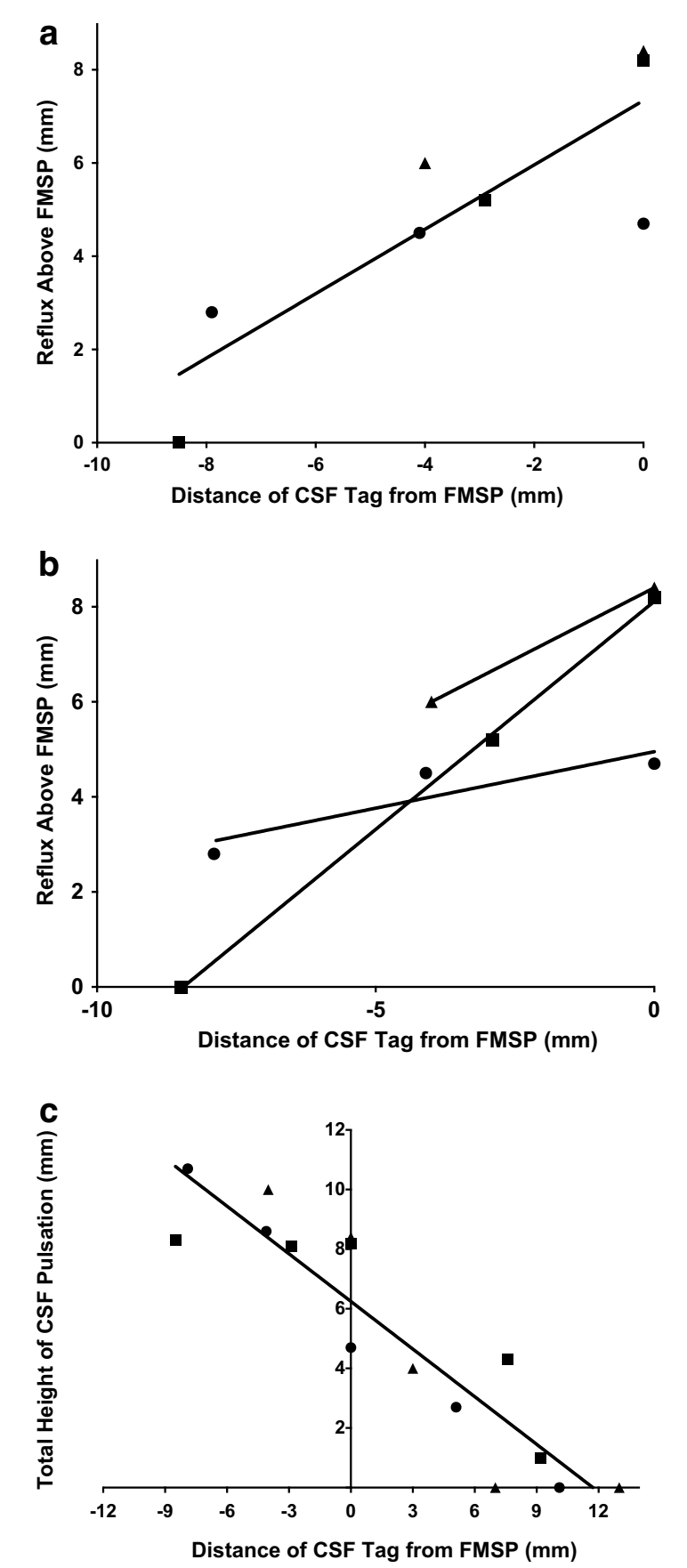

Figure 1 a Effect of CSF tag position on the amount of visualized CSF reflux from the 3 rd ventricle into the lateral ventricles. Measurements are relative to the plane of the free margin of the septum pellucidum (FMSP). As the tag position is situated closer to the FMSP, there is an increase in visualized CSF reflux $(P=0.006)$. $\mathbf{b}$ The effect of CSF tag position on the amount of visualized CSF reflux on an individual subject level. The same effect of increasing reflux as the tag is moved closer to the FMSP is illustrated in each individual subject. c Effect of CSF tag position on the height of visualized CSF pulsation above the tag. Higher CSF pulsation is seen as the tag position is moved caudally $(P<0.0001)$. Triangle subject 1 , square subject 2 , circle subject 3 . confirmed a progressive decrease in visualized reflux with increasing distance from the FMSP within each subject (Figure 1b). We found no significant measurable difference between maximum reflux height observed between the right and left sides of the brain across all measurements.

The position of the tag also had a measurable effect on the visualized total height of CSF pulsation (Figure 1c). As the tag was moved caudally from a position originating in the upper aspect of the lateral ventricles, the total height of CSF pulsation above the tag increased $\left(\mathrm{y}=0.53 \mathrm{x}+6.2 ; \mathrm{R}^{2}=0.84 ; P<0.0001\right)$.

Despite increasing height of pulsation as the tag is moved caudally, there continued to be a decrease in the amount of observed reflux into the lateral ventricles (Figure 2). We identified reflux in some instances with the tag placed well below the FMSP. However, it is evident that the reflux was underestimated as each subject had decreasing reflux as the distance from the FMSP increased (Figure 1b).

\section{Discussion}

The results of our study show a substantial variation in the amount of observed reflux from the third ventricle into the lateral ventricles based upon the position of the CSF tag in Time-SLIP imaging. For demonstration of reflux into the lateral ventricles, we found that the optimum tag location is with the top (superior) border of the tag at the level of the FMSP.

We postulate the explanation of these findings is that the majority of CSF exchanged between the third and lateral ventricles during normal pulsation is from fluid located in the cephalad portion of the third ventricle. Fluid tagged in the more caudal portion of the third ventricle contributes less to the overall exchanged pool of fluid. An alternative hypothesis would be that fluid closer to the foramen experiences a higher reflux velocity resulting in an apparent increase in reflux height.

Modern investigation into the normal flow of CSF has suggested a much more complex circulation than was historically thought [12-15]. In vivo imaging techniques have promoted the idea of a pulsatile nature of CSF flow as opposed to the classic theory of bulk flow from the choroid plexus of the ventricular system outward into the subarachnoid space $[13,16]$. The pulsatile nature results in bidirectional flow through the ventricular system which can drive fluid from the subarachnoid space back as far as the lateral ventricles [17]. This system drives the regulation of intracranial pressure and the clearance of metabolites, many of which are pro-inflammatory or toxic to the brain substance $[1,2]$. Since the brain lacks true lymphatic vasculature, the majority of interstitial fluid clearance is through the CSF $[1,2]$.

Disturbance of the normal pulsatile nature of CSF flow has been shown to correlate with many diseases 

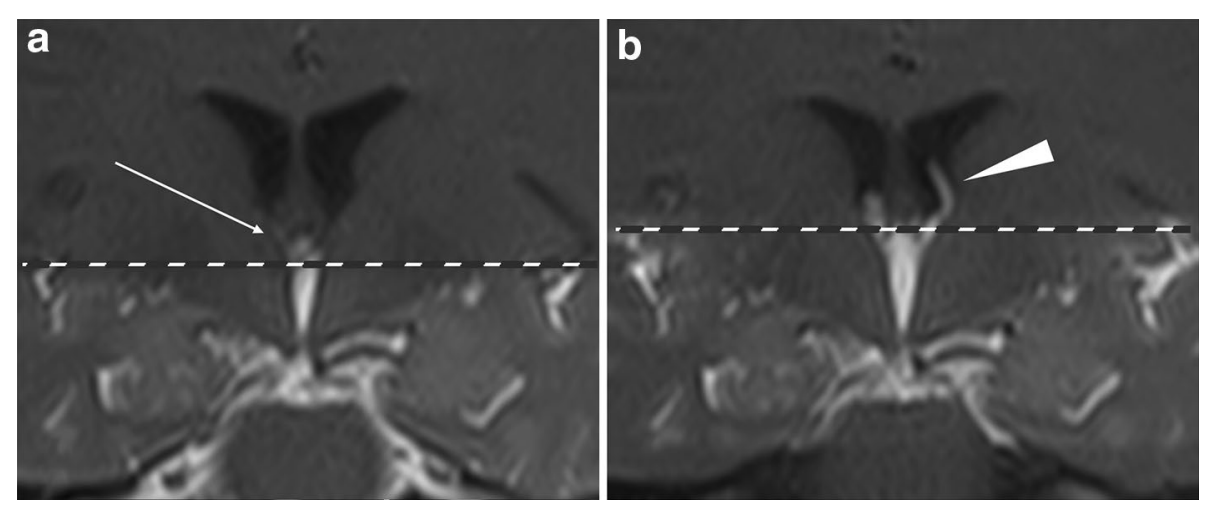

Figure 2 Coronal Time-SLIP MR images in the same subject illustrating the effect of improper CSF tag placement. a When the top of the tag (dashed line) was placed more inferior to the free margin of the septum pellucidum (FMSP), minimal CSF reflux (arrow) was seen into the lateral ventricles. b Once the top of the tag (dashed line) was moved to the plane of the FMSP, reflux was easily identified into the lateral ventricles (arrowhead).

of the CNS. Recent studies have confirmed decreased reflux from the third ventricle into the lateral ventricles in conditions such as aqueductal stenosis [18], noncommunicating hydrocephalus [9], and normal pressure hydrocephalus [11]. It is also possible that alterations in normal CSF physiology play a role in the pathogenesis of other diseases of the CNS. The application of techniques described in this study may prove useful in further elucidating both the normal physiologic flow of CSF, as well as in assessment of underlying pathologic disturbances.

The advent of MRI sequences sensitive to flow effects has greatly advanced our understanding of normal and abnormal CSF flow patterns. One of the most commonly used MRI pulse sequences is phase-contrast imaging (PCMRI) [7, 8]. Phase-contrast MRI relies on the detection of complex phase changes in moving fluid [7]. An advantage of PC-MRI is the ability to quantify CSF flow [7, 11]. A primary disadvantage of PC-MRI, however, is that turbulent CSF flow $[11,19,20]$ and bulk CSF flow $[11,21]$ cannot be reliably visualized. Time-SLIP imaging allows the noninvasive visualization of CSF displacement without relying on velocity-dependent phase changes. The principle underlying time-SLIP imaging is visualization of fluid displacement by selective magnetic tagging of protons. This technique can be applied to visualize the amount of fluid displacement from the third ventricle into the lateral ventricles. The bidirectional flow through the foramen of Monro related to the cardiac and respiratory cycle results in the mixing of tagged protons in the third ventricular CSF refluxing back into the lateral ventricles. This technique allows improved characterization of turbulent flow and CSF stasis relative to phase-contrast techniques.

We have shown that the amount of visualized CSF reflux into the lateral ventricles in Time-SLIP imaging is dependent on the positioning of the CSF tag. To the authors' knowledge, there are no existing publications describing the distance dependence of Time-SLIP tag placement on the amount of visualized CSF reflux. Our results suggest caution be exercised when interpreting a lack of reflux as pathologic when the tag position is not optimal. We have also illustrated that inappropriate tag positioning may continue to show CSF reflux, but the amount of reflux will be underestimated. The information presented herein is essential for future studies assessing the physiologic and pathologic variability of this phenomenon.

\section{Conclusion}

The amount of observed CSF reflux into the lateral ventricles in Time-SLIP imaging is dependent on the positioning of the CSF tag with decreasing amount of reflux the further caudal the CSF tag is relative to the free margin of the septum pellucidum.

\section{Additional files}

Additional file 1: Figure S1. MR images showing the selection of imaging plane. (A) Sagittal localizer illustrates the location and orientation of the oblique coronal imaging plane (line) which is parallel to the foramen of Monro (arrowhead). (B) Coronal localizer demonstrating the ideal plane for illustration of reflux of CSF from the third ventricle into the lateral ventricles. The coronal oblique plane is chosen to visualize the foramen of Monro (arrow).

Additional file 2: Figure S2. (A) MR images showing the plane of the free margin of the septum pellucidum (line) perpendicular to the free margin of the septum pellucidum (arrow). (B) Normal reflux into the lateral ventricle is demonstrated (arrow).

Additional file 3: Figure S3. Measurement of CSF pulsation. Example of recorded measurements including measurement of the total height of CSF pulsation (larger double-headed arrow) above the CSF tag (dashed line) and height of CSF reflux into lateral ventricle above the plane of the free margin of the septum pellucidum (small double-headed arrow). 


\section{Abbreviations}

Time-SLIP: time-spatial labeling inversion pulse; CSF: cerebrospinal fluid; PCMRI: phase-contrast magnetic resonance imaging; FMSP: free margin of the septum pellucidum.

\section{Authors' contribution}

$E M, J B$, and $A O C$ conceived and designed the study. EM and AOC carried out the imaging studies. JB and EM measured CSF movement on the acquired imaging. EM performed the statistical analysis. EM drafted the manuscript. JB and $A O C$ edited and revised the manuscript. All authors read and approved the final manuscript.

\section{Author details}

${ }^{1}$ Department of Radiology, College of Medicine, University of Florida, 1600 SW Archer Rd., PO Box 100374, Gainesville, FL 32610, USA. ${ }^{2}$ K. Scott and E.R. Andrew Advanced Neuroimaging Lab, University of Florida, Gainesville, FL, USA.

\section{Acknowledgements}

The authors thank Jonathan Furuyama for technical assistance with the pulse sequence.

\section{Compliance with ethical guidelines}

\section{Competing interests}

The authors declare that they have no competing interests.

Received: 23 April 2015 Accepted: 10 June 2015

Published online: 21 June 2015

\section{References}

1. Iliff JJ, Wang M, Liao Y, Plogg BA, Peng W, Gundersen GA, et al. A paravascular pathway facilitates CSF flow through the brain parenchyma and the clearance of interstitial solutes, including amyloid beta. Sci Transl Med. 2012:4(147):147ra111.

2. Abbott NJ. Evidence for bulk flow of brain interstitial fluid: significance for physiology and pathology. Neurochem Int. 2004;45(4):545-52.

3. Luetmer PH, Huston J, Friedman JA, Dixon GR, Petersen RC, Jack CR, et al. Measurement of cerebrospinal fluid flow at the cerebral aqueduct by use of phase-contrast magnetic resonance imaging: technique validation and utility in diagnosing idiopathic normal pressure hydrocephalus. Neurosurgery. 2002;50(3):534-43.

4. Bateman GA. The role of altered impedance in the pathophysiology of normal pressure hydrocephalus. Alzheimer's disease and syringomyelia. Med Hypotheses. 2004;63(6):980-5.

5. Haughton V, Mardal KA. Spinal fluid biomechanics and imaging: an update for neuroradiologists. Am J Neuroradiol. 2014;35(10):1864-9.

6. Gorucu Y, Albayram S, Balci B, Hasiloglu ZI, Yenigul K, Yargic F, et al. Cerebrospinal fluid flow dynamics in patients with multiple sclerosis: a phase contrast magnetic resonance study. Funct Neurol. 2011;26(4):215-22.

7. Feinberg DA, Mark AS. Human brain motion and cerebrospinal fluid circulation demonstrated with MR velocity imaging. Radiology. 1987;163(3):793-9.
8. Battal B, Kocaoglu M, Bulakbasi N, Husmen G, Tuba Sanal H, Tayfun C. Cerebrospinal fluid flow imaging by using phase-contrast MR technique. Br J Radiol. 2011;84(1004):758-65.

9. Yamada S, Miyazaki M, Kanazawa H, Higashi M, Morohoshi Y, Bluml S, et al. Visualization of cerebrospinal fluid movement with spin labeling at MR imaging: preliminary results in normal and pathophysiologic conditions. Radiology. 2008;249(2):644-52.

10. Yamada S. Cerebrospinal fluid physiology: visualization of cerebrospinal fluid dynamics using the magnetic resonance imaging time-spatial inversion pulse method. Croat Med J. 2014;55(4):337-46.

11. Yamada S, Tsuchiya K, Bradley WG, Law M, Winkler ML, Borzage MT, et al. Current and emerging mr imaging techniques for the diagnosis and management of CSF flow disorders: a review of phase-contrast and timespatial labeling inversion pulse. Am J Neuroradiol. 2015;36(4):623-30.

12. Greitz D. Paradigm shift in hydrocephalus research in legacy of Dandy's pioneering work: rationale for third ventriculostomy in communicating hydrocephalus. Childs Nerv Syst. 2007;23(5):487-9.

13. Greitz D. Cerebrospinal fluid circulation and associated intracranial dynamics. A radiologic investigation using MR imaging and radionuclide cisternography. Acta Radiol Suppl. 1993;386:1-23.

14. Greitz D, Greitz T, Hindmarsh T. A new view on the CSF-circulation with the potential for pharmacological treatment of childhood hydrocephalus. Acta Paediatr Suppl. 1997;86(2):125-32.

15. Greitz D, Hannerz J. A proposed model of cerebrospinal fluid circulation: observations with radionuclide cisternography. Am J Neuroradiol. 1996;17(3):431-8.

16. Greitz D, Franck A, Nordell B. On the pulsatile nature of intracranial and spinal CSF-circulation demonstrated by MR imaging. Acta Radiol. 1993:34(4):321-8.

17. Hladky SB, Barrand MA. Mechanisms of fluid movement into, through and out of the brain: evaluation of the evidence. Fluids Barriers CNS. 2014;11(1):26.

18. Abe $K$, Ono $Y$, Yoneyama $H$, Nishina $Y$, Aihara $Y$, Okada $Y$, et al. Assessment of cerebrospinal fluid flow patterns using the time-spatial labeling inversion pulse technique with 3T MRI: early clinical experiences. Neuroradiol J. 2014;27(3):268-79.

19. Sherman JL, Citrin CM, Gangarosa RE, Bowen BJ. The MR appearance of CSF flow in patients with ventriculomegaly. Am J Roentgenol. 1987;148(1):193-9.

20. Malko JA, Hoffman JC Jr, McClees EC, Davis PC, Braun IF. A phantom study of intracranial CSF signal loss due to pulsatile motion. Am J Neuroradiol. 1988;9(1):83-9.

21. Wagshul ME, Chen JJ, Egnor MR, McCormack EJ, Roche PE. Amplitude and phase of cerebrospinal fluid pulsations: experimental studies and review of the literature. J Neurosurg. 2006;104(5):810-9.

\section{Submit your next manuscript to BioMed Central and take full advantage of:}

- Convenient online submission

- Thorough peer review

- No space constraints or color figure charges

- Immediate publication on acceptance

- Inclusion in PubMed, CAS, Scopus and Google Scholar

- Research which is freely available for redistribution 